\title{
Perfil de Uso das Ferramentas de Internet por Alunos de Licenciatura em Biologia na Modalidade Semipresencial
}

\author{
Neusa Helena da Silva Pires Martins*1, Daniel Fábio Salvador*2, Luiz Gustavo \\ Ribeiro Rolando*3, Maurício Roberto Motta Pinto da Luz*4.
}

\footnotetext{
1 Mestranda, Laboratório Avaliação em Ensino e Filosofia das Biociências /Instituto Oswaldo Cruz/Fundação Oswaldo Cruz. Av. Brasil, 4365, Manguinhos - Rio de Janeiro - RJ - Brasil. nhdsp@ioc.fiocruz.br

2 Pesquisador, Departamento de Mídias Digitais/ Fundação CECIERJ. Rua da Ajuda, n 5 - Rio de Janeiro RJ - País. salvador@cecierj.edu.br

3 Doutorando, Laboratório Avaliação em Ensino e Filosofia das Biociências /Instituto Oswaldo Cruz/Fundação Oswaldo Cruz. Av. Brasil, 4365, Manguinhos - Rio de Janeiro - RJ - Brasil. gustavor@ioc.fiocruz.br

${ }^{4}$ Pesquisador, Laboratório Avaliação em Ensino e Filosofia das Biociências /Instituto Oswaldo Cruz/Fundação Oswaldo Cruz. Av. Brasil, 4365, Manguinhos - Rio de Janeiro - RJ - Brasil. mauluz@ioc.fiocruz.br
}

\section{Resumo}

Para criar um ambiente adequado à aprendizagem de Biologia por meio das ferramentas da internet, é fundamental conhecer as necessidades dos alunos. Isso é possível ao investigar o perfil de uso dessas ferramentas por estudantes de Biologia. Para isso, foi aplicado um questionário com perguntas abertas e fechadas sobre a frequência e o modo de uso das ferramentas. Por meio desse instrumento, vê-se que os alunos utilizam pouco as ferramentas de interação da Web 2.0. Porém, o uso do Facebook tem se mostrado como uma ferramenta positiva para aprendizagem além dos conteúdos formais, mostrando a necessidade de se pensar estratégias voltadas para levar os alunos à utilização das potencialidades das redes sociais pouco exploradas no Brasil.

Palavras-chave: Educação a distância; Aprendizagem baseada na web; Ferramentas da internet; Tecnologias da informação e comunicação. 


\title{
Profile Using Internet Tools for Biology Degree Students in Blended Mode
}

\begin{abstract}
To create a suitable environment for learning biology through Internet tools it is vital to know the needs of the students. This is possible if the profile of use of these tools for biology students is investigated. To investigate, a questionnaire with open and closed questions about the frequency and use mode of the tools was applied. Through this tool we can see that students rarely use the interactive tools of the Web 2.0. However, the use of Facebook has proven to be a positive tool for learning beyond the formal contents, showing the need to think about strategies for teaching pupils to use the potential of social networking that is less explored in Brazil.
\end{abstract}

Keywords: distance education; Web based learning; Internet tools; Information and communications technology. 


\section{Introdução}

O uso de ferramentas da internet para auxiliar no processo de ensino e aprendizagem vem crescendo a cada dia. Atualmente, as novas tecnologias possibilitam que qualquer participante possa ser um criador de conteúdo, diferentemente da Web 1.0, cujas funcionalidades estavam voltadas para o seu consumo (Cormode \& Krishnamurthy, 2008). O conteúdo hoje pode ser criado e compartilhado colaborativamente por qualquer pessoa sem a necessidade de conhecimentos em programação ou WEB Design. Assim, qualquer indivíduo com habilidade mínima nesses recursos pode se tornar produtor de informação, contribuindo para o crescimento do conteúdo disponibilizado na web. (Brown, 2012).

Ao contribuir nesse espaço, o indivíduo deixa de ser um mero observador para se tornar um colaborador e criador do conhecimento. Com isso, as tecnologias emergentes conduzem a possibilidades, inimagináveis até certo tempo atrás, para fomentar a aprendizagem (Bransford, Brow, \& Cocking, 2007).

Gialamas, Nikolopoulou e Koutromanos (2013) demonstraram em seu trabalho que a percepção de estudantes de educação infantil sobre o impacto da internet para sua aprendizagem e trabalhos futuros é positiva. Ullrich et al. (2008) apontam que a exploração da Web 2.0 tem um grande potencial para a aprendizagem apoiada por tecnologias, mas é necessário ter como foco principal as necessidades do aluno.

A utilização da Web 2.0 tem permitido a expansão das possibilidades pedagógicas no uso da internet, porém seu uso ainda é pouco explorado para fins didáticos por docentes no Brasil (Rolando, Salvador e Luz, 2013). Entende-se como Web 2.0 o conjunto de serviços da internet que propiciam ao usuário a construção de conhecimento na rede de forma colaborativa por meio de ferramentas de interação. O'reilly (2007) descreve a Web 2.0 como a web em plataforma, abrangendo todos os dispositivos conectados. Para ele, a principal competência presente nos aplicativos da Web 2.0 é a potencialidade de explorar as contribuições do usuário para a rede, aproveitando da inteligência coletiva.

No Brasil, a busca da utilização dessas tecnologias para a aprendizagem vem sendo fomentada por vários documentos oficiais, inclusive ressaltando a importância de 
contemplar as Tecnologias da Informação e Comunicação (TIC) na formação para atividade docente (Resolução CNE/CP no 1/2002; CONAE, 2010 \& FNDE, 2010).

Os futuros professores devem adquirir conhecimentos para que possam utilizar as TIC tanto nos processos de aprendizagem na licenciatura quanto na sua vida profissional (Yapici \& Hevedanli, 2012).

Alguns cursos de formação docente têm oferecido disciplinas voltadas à capacitação dos licenciandos no uso das tecnologias para suas futuras práticas docentes. Porém, o processo para desmistificar a utilização das tecnologias para os futuros professores inserindo conceitos da Web 2.0, das TIC e da Aprendizagem Colaborativa com Suporte Computacional ainda é pouco explorado.

A Aprendizagem Colaborativa com Suporte Computacional (do inglês Computersupported collaborative learning - CSCL) tem por base o uso de ferramentas computacionais para promover a aprendizagem colaborativa (Stahl, Koschmann, \& Suthers, 2006). Na aprendizagem colaborativa, duas ou mais pessoas podem aprender ou tentar aprender sobre algo juntas, presencialmente ou por suporte computacional (Dillenbourg, 1999).

O uso de redes sociais para interação e comunicação entre estudantes de graduação tem aumentado cada dia mais, sendo o Facebook o meio preferido por eles (Hussain 2012). Por meio dessa ferramenta, eles podem estabelecer uma comunicação síncrona ou assíncrona possibilitando que aprendam colaborativamente.

Mas, para se criar um ambiente adequado de aprendizagem sobre o uso desses recursos, torna-se necessário entender como os alunos utilizam as ferramentas da internet para estudo, quais potencialidades já foram atingidas e o quanto elas contribuem não só para sua formação, mas também para seu desenvolvimento profissional.

O objetivo desse estudo é investigar como os alunos de um curso de licenciatura em ciências biológicas semipresencial utilizam as ferramentas da internet para aprender e estudar Biologia. 


\section{Metodologia}

\subsection{Contexto do Estudo}

O presente estudo foi realizado com alunos de um curso de licenciatura em Ciências Biológicas da Fundação CECIERJ/Consórcio CEDERJ na modalidade semipresencial no Estado do Rio de Janeiro. O curso é oferecido em 19 polos regionais distribuídos pelo Estado e oferece, em sua grade curricular, disciplinas obrigatórias e eletivas que contam com avaliações presenciais e a distância.

Durante a graduação, os licenciandos podem optar por cursar a disciplina Tecnologias Educacionais para o Ensino de Biologia e Ciências. A disciplina é oferecida semestralmente e todas as suas aulas e avaliações são a distância, ofertadas por meio da plataforma educacional Moodle (Modular Object-Oriented Dynamic Learning Environment). Por ser uma disciplina eletiva, qualquer aluno, a partir do $5^{\circ}$ período da graduação, pode cursá-la. Seu objetivo é a formação didático-tecnológica do licenciando em Ciências Biológicas para o uso da internet e suas ferramentas, apresentando suas possibilidades e aplicações didáticas.

\subsection{Amostra}

Participaram do estudo 254 licenciandos regularmente matriculados no curso de licenciatura em Ciências Biológicas e que cursaram a disciplina Tecnologias Educacionais para o Ensino de Biologia e Ciências (TECBIO) durante o ano de 2013.

\subsection{Instrumento de Coleta de Dados}

Na primeira semana da disciplina de TECBIO, os alunos responderam a um questionário (Apêndice 1) com 34 perguntas. Esse questionário foi adaptado do instrumento utilizado e validado por Rolando, Salvador e Luz (2013). Foram abordadas as seguintes ferramentas da internet: Sites de Busca, E-mail, Chat, Twitter, Skype, Download, Imagens, Vídeos, Wikipédia, Google Docs, Blogs, Fóruns, Facebook, Orkut, Google +, Ning, Second Life.

As perguntas fechadas buscaram identificar a frequência semanal de uso das ferramentas supracitadas com possibilidades de respostas que variavam do "Não utilizo" até o uso "7 vezes por semana". 
Já as perguntas abertas tiveram por objetivo explorar as finalidades com as quais os licenciandos utilizam as ferramentas da internet para estudar e aprender Biologia.

O procedimento para a análise dos dados obtidos por meio das respostas às perguntas abertas foi realizado utilizando-se metodologia qualitativa. Para encontrar características comuns seguiu-se o processo de análise de conteúdo no qual os dados foram identificados, nomeados e codificados (Fraenkel, \& Wallen, 2008). As respostas foram classificadas em cinco categorias, a saber: Atividades da Graduação, Dúvidas, Aprofundamento de conteúdo, Conteúdos Biológicos em Geral e Não informativo (Quadro 1). As respostas classificadas como não informativas foram excluídas das análises, uma vez que não contribuem para o entendimento das finalidades de uso das ferramentas. Além disso, embora as categorias sejam mutuamente excludentes, uma mesma ferramenta pode ter sido utilizada para diferentes finalidades. Dessa forma, uma mesma resposta aberta pode ter contribuído para mais de uma categoria, gerando, com isso, um número de categorizações superior ao número de respostas.

Quadro 1: Descrição das categorias que definem a finalidade de uso das ferramentas da internet.

\begin{tabular}{|c|c|c|}
\hline Categorias & Descrição & Exemplos \\
\hline $\begin{array}{l}\text { I - Atividades } \\
\text { da Graduação }\end{array}$ & $\begin{array}{l}\text { Uso das ferramentas para atender a } \\
\text { uma atividade proposta em alguma } \\
\text { disciplina do seu curso. }\end{array}$ & $\begin{array}{l}\text { "Para fazer avaliações a } \\
\text { distância e relatórios" }\end{array}$ \\
\hline II - Dúvidas & $\begin{array}{l}\text { Uso das ferramentas para tirar } \\
\text { dúvidas com relação a conteúdos } \\
\text { biológicos. }\end{array}$ & $\begin{array}{l}\text { "Faço buscas de acordo com o } \\
\text { aparecimento de dúvidas nas } \\
\text { disciplinas" }\end{array}$ \\
\hline $\begin{array}{l}\text { III } \\
\text { Aprofunda- } \\
\text { mento de } \\
\text { conteúdo }\end{array}$ & $\begin{array}{l}\text { Uso das ferramentas para aprofundar } \\
\text { o entendimento acerca de conteúdos } \\
\text { biológicos que estão sendo abordados } \\
\text { no curso de graduação. }\end{array}$ & $\begin{array}{l}\text { "Utilizo para complementação } \\
\text { do estudo" "Acho uma ótima } \\
\text { maneira de complementar } \\
\text { informações e ir mais longe } \\
\text { nos estudos" }\end{array}$ \\
\hline $\begin{array}{l}\text { IV - } \\
\text { Conteúdos } \\
\text { Biológicos em } \\
\text { Geral }\end{array}$ & $\begin{array}{l}\text { Uso das ferramentas para ter acesso } \\
\text { a conteúdos biológicos que não estão } \\
\text { sendo abordados no curso de } \\
\text { graduação. }\end{array}$ & 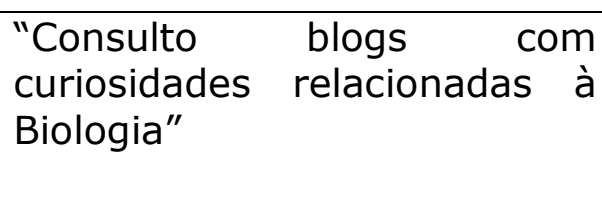 \\
\hline $\begin{array}{l}\text { V } \quad \text { Não } \\
\text { informativo }\end{array}$ & $\begin{array}{l}\text { As respostas não possibilitaram uma } \\
\text { categorização. }\end{array}$ & Não se aplica \\
\hline
\end{tabular}


Para a ferramenta downloads foram contabilizadas as respostas para oito tipos de arquivos: Artigos científicos, Textos, Vídeos, Slides, Teses, dissertações e monografias, Imagens, Avaliações do curso e Jogos.

Na ferramenta Wikipédia, foram contabilizadas as respostas relativas à sua confiabilidade. Quando o participante emitia uma opinião relativa à confiabilidade da ferramenta, esta era classificada em "Confiável" ou "Não Confiável".

Além disso, as respostas abertas para as ferramentas da internet, quando possível, eram classificadas quanto ao tipo de uso em conteúdos do curso ou conteúdos fora do curso.

\section{Resultados e Discussão}

As perguntas fechadas foram contabilizadas para determinar a frequência de uso de cada uma das ferramentas da internet pelos licenciandos (Figura 1). O total de respostas quanto às perguntas fechadas, direcionadas à regularidade do uso das ferramentas, foi de 4318 , e todos os participantes foram direcionados a escolher uma opção, variando de "Não" até "7 dias por semana". Os dados demonstram que as ferramentas utilizadas mais frequentemente pelos alunos são os Sites de Busca (mais de $40 \%$ dos participantes os utilizam até cinco vezes por semana), Download e E-mail (aproximadamente $25 \%$ de uso até cinco vezes por semana).

Nota-se que o uso das ferramentas voltadas para o consumo da informação é mais frequente pelos alunos. Já as ferramentas que propiciam a criação de conteúdo são pouco utilizadas, exceto o Facebook.

Com relação ás redes sociais, vê-se que o Facebook é a mais utilizada, pois cerca de $40 \%$ dos licenciandos a acessa pelo menos três vezes na semana para estudar Biologia. Hussain (2012) demonstrou a preferência no uso dessa ferramenta por estudantes do Departamento de Educação da Faculty of Education of the Islamia University of Bahawalpur para interação. Outro ponto a se relatar é o baixo uso do Twitter pelos participantes, já que mais de $90 \%$ não o utilizam como ferramenta para o estudo de Ciências Biológicas. 


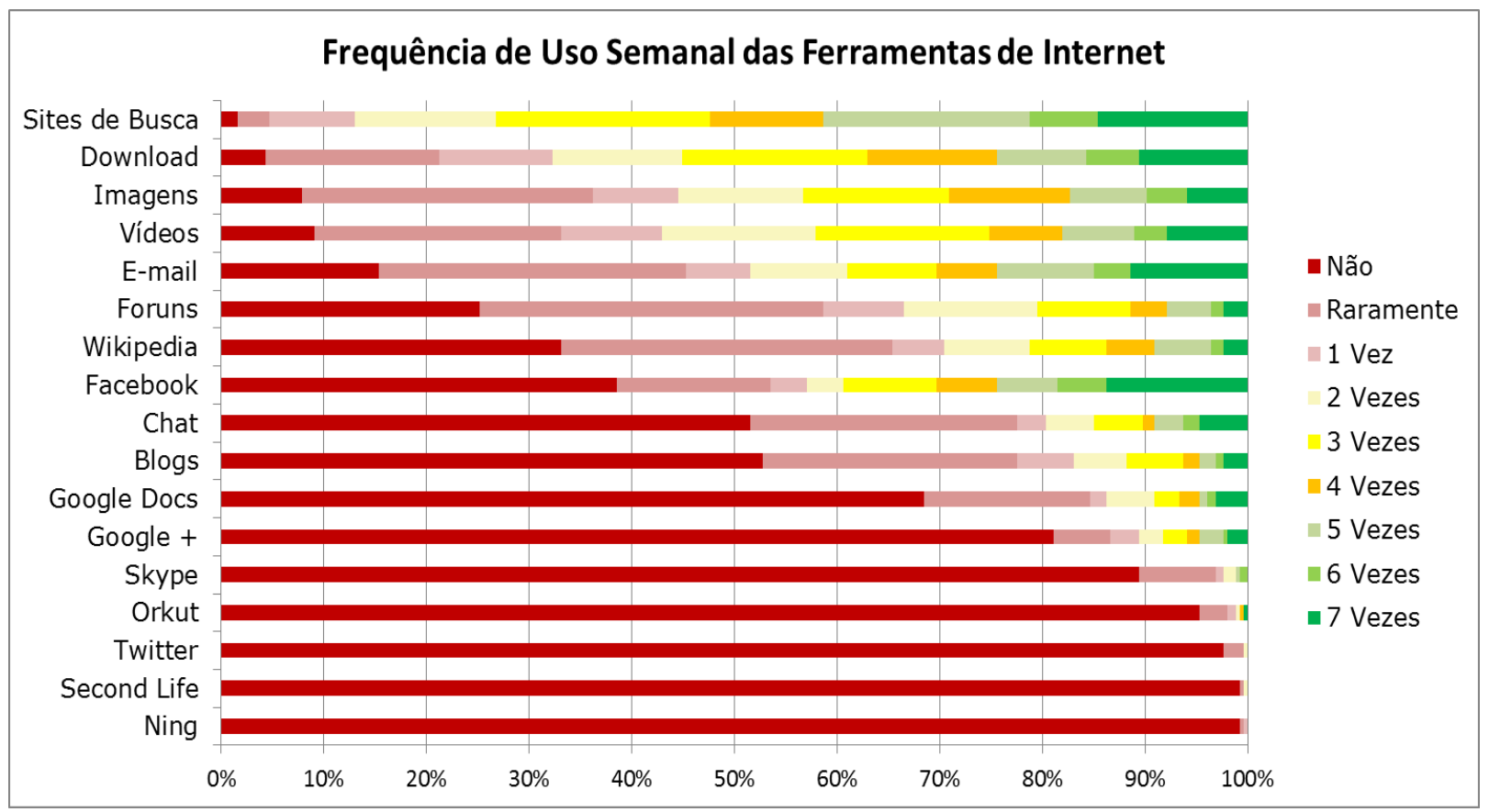

Figura 1 - Frequência de uso semanal das ferramentas de internet por alunos de licenciatura em ciências biológicas na modalidade semipresencial ( $n=4318$ respostas).

Com base nas categorias que emergiram das respostas abertas, foi traçado o percentual da utilização das ferramentas de um total de 2133 categorizações. As duas finalidades de uso mais comuns são a realização de atividades do curso de graduação (24\%) e o aprofundamento de conteúdo do curso (23\%).

A categoria aprofundamento de conteúdo, apesar de estar intrinsecamente ligada à graduação, propicia um olhar sobre o quanto as ferramentas têm auxiliado os alunos a ter uma visão mais ampla dos assuntos, permitindo assim encontrar diferentes pontos de vistas de uma mesma informação.

Com relação às dúvidas no curso, 13\% das ferramentas são utilizadas para atender a essa demanda. Já se tratando da categoria de Conteúdos Biológicos em Geral, cerca de $14 \%$ do uso está direcionado a essa finalidade, o que mostra que os alunos têm usado as ferramentas de internet para aprender conteúdos biológicos externos ao seu curso.

O uso das ferramentas voltado para conteúdos externos ao curso pode contribuir para uma aprendizagem com o uso das TIC para além da licenciatura, auxiliando na formação profissional, como apontado por Yapici \& Hevedanli (2012). Por meio desses recursos, os licenciandos se mantêm informados sobre a área sem que sejam 
direcionados por suas disciplinas, optando por quais conteúdos são realmente relevantes para sua aprendizagem.

As redes sociais e os blogs se destacam como as ferramentas mais utilizadas pelos participantes na categoria de Conteúdos Biológicos em Geral (Tabela 1).

Tabela 1 - Finalidades de uso das Ferramentas da internet por alunos de licenciatura em ciências biológicas.

Tabela de Categorização

\begin{tabular}{|c|c|c|c|c|c|}
\hline Ferramenta & $\mathbf{N}$ & $\begin{array}{c}\text { Atividades } \\
\text { da } \\
\text { graduação }\end{array}$ & Dúvidas & $\begin{array}{l}\text { Aprofunda- } \\
\text { mento de } \\
\text { conteúdo }\end{array}$ & $\begin{array}{c}\text { Conteúdos } \\
\text { biológicos em } \\
\text { Geral }\end{array}$ \\
\hline Sites de Busca & 248 & $26 \%$ & $28 \%$ & $29 \%$ & $17 \%$ \\
\hline Imagens & 222 & $25 \%$ & $8 \%$ & $56 \%$ & $12 \%$ \\
\hline Vídeos & 215 & $22 \%$ & $11 \%$ & $49 \%$ & $17 \%$ \\
\hline Fóruns & 166 & $73 \%$ & $14 \%$ & $7 \%$ & $5 \%$ \\
\hline E-mail & 147 & $37 \%$ & $32 \%$ & $10 \%$ & $21 \%$ \\
\hline Download & 138 & $38 \%$ & $3 \%$ & $45 \%$ & $14 \%$ \\
\hline Facebook & 106 & $29 \%$ & $22 \%$ & $3 \%$ & $46 \%$ \\
\hline Chat & 92 & $43 \%$ & $41 \%$ & $11 \%$ & $4 \%$ \\
\hline Wikipédia & 90 & $17 \%$ & $20 \%$ & $50 \%$ & $13 \%$ \\
\hline Blogs & 75 & $8 \%$ & $4 \%$ & $27 \%$ & $61 \%$ \\
\hline Google Docs & 21 & $48 \%$ & $5 \%$ & $33 \%$ & $14 \%$ \\
\hline Google + & 12 & $17 \%$ & $8 \%$ & $33 \%$ & $42 \%$ \\
\hline Skype & 11 & $45 \%$ & $55 \%$ & - & - \\
\hline Twitter & 5 & - & $20 \%$ & - & $80 \%$ \\
\hline Ning & 1 & - & - & - & $100 \%$ \\
\hline Orkut & 0 & - & - & - & - \\
\hline Second Life & 0 & - & - & - & - \\
\hline Total & 1549 & $33 \%$ & $18 \%$ & $31 \%$ & $19 \%$ \\
\hline
\end{tabular}


Os sites de busca têm uma distribuição homogênea entre as categorias, não havendo prevalência expressiva em nenhuma delas. Quanto ao uso dos fóruns, a prevalência de seu uso está destinada às atividades de graduação, o que é uma característica típica dessa modalidade de ensino, que utiliza essa ferramenta para realização de exercícios.

Outro fator a se destacar do comportamento dos participantes é a utilização de ferramentas que permitem trocas por meio de videoconferência, como chats e Skype, ligadas principalmente ao curso, com mais de $80 \%$ do seu uso destinado ás atividades da graduação e dúvidas nas disciplinas.

Algumas ferramentas da internet permitiram uma observação complementar, como o Download e a Wikipédia. Com relação ao tipo de arquivos em que os alunos realizam downloads, temos que de 251 respostas abertas que possibilitaram a identificação dos arquivos, 32\% citam que seus downloads são de artigos científicos, $27 \%$ de textos diversos, $14 \%$ de vídeos, $10 \%$ de imagens e $6 \%$ de teses, dissertações e monografias. Apesar de revelar uma preocupação com a qualidade das informações que são utilizadas por esses alunos, ao utilizarem artigos científicos primariamente, o uso de textos de lugares variados como fontes de pesquisa ainda está presente em sua prática, enquanto as teses, dissertações e monografias que são boas fontes de informação têm uma baixa procura.

Um total de 170 alunos afirmou que utiliza Wikipédia como uma fonte de pesquisa. Em 57 respostas abertas, que permitiram tal análise de confiança, encontramos que $93 \%$ afirmam que não confiam no conteúdo apresentado embora a utilizem. Os licenciandos citam em suas declarações que esse uso é destinado ao primeiro contato com o conteúdo a ser pesquisado e que, após compreenderem o assunto, realizam buscas mais aprofundadas em sites que consideram mais seguros.

A caracterização do uso das ferramentas, como voltadas para conteúdos relacionados ao seu curso ou de fora do curso, temos que a maior parte do uso destinase ao curso (Figura 2). 


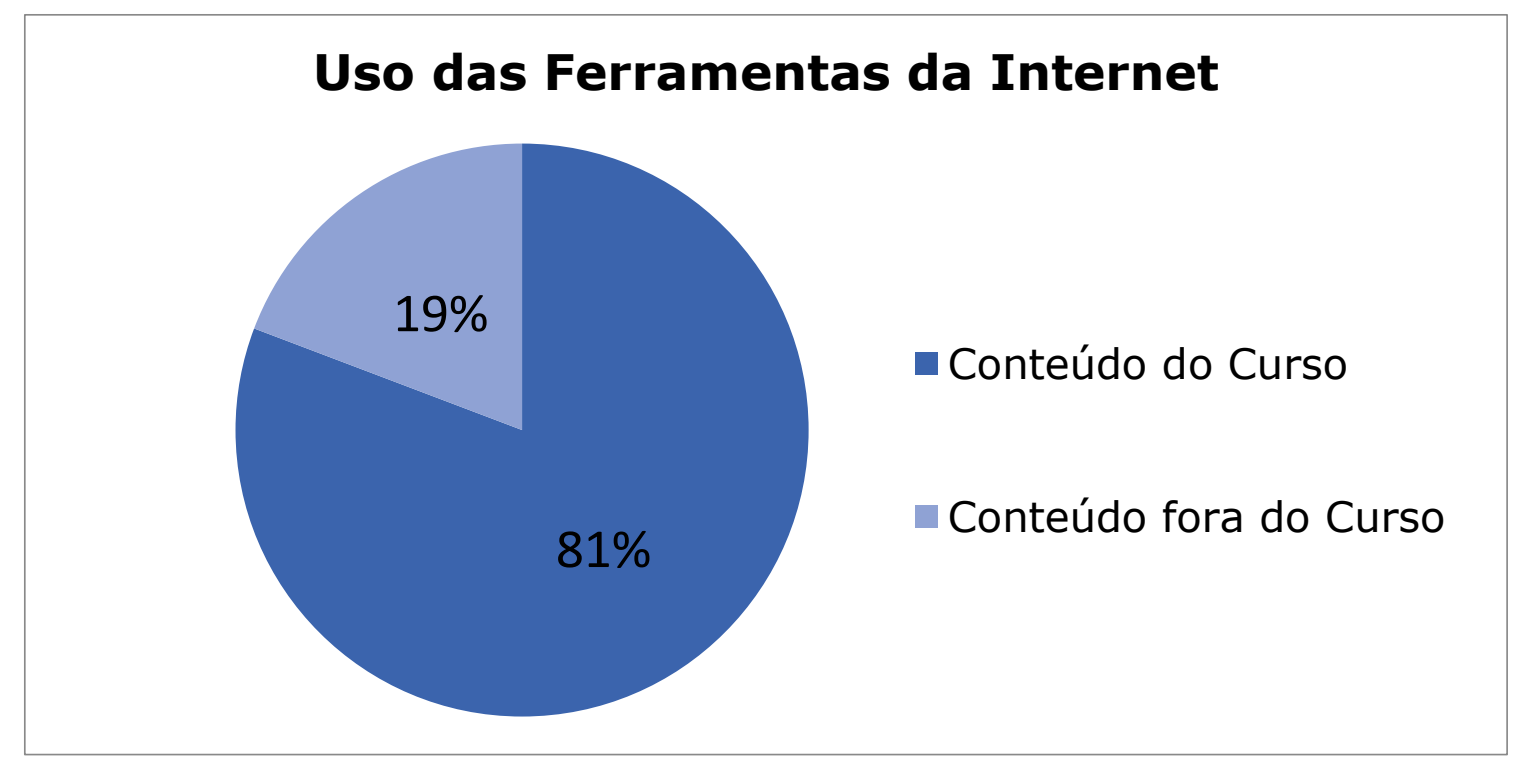

Figura 2 - Caracterização do uso das ferramentas como conteúdos do curso e conteúdos fora do curso. ( $n=1170$ respostas abertas)

Isso mostra que esses alunos têm direcionado o uso das ferramentas da internet para atender a demandas com relação a sua graduação, uma vez que se trata de um curso semipresencial. Contudo, a aplicação das ferramentas para assuntos além dos trabalhados em suas disciplinas é um fator positivo, pois demonstra que as ferramentas da internet podem contribuir para uma aprendizagem centrada nas necessidades dos alunos e não direcionada apenas por um currículo.

\section{Considerações finais}

Com base no presente levantamento, foi verificado que os alunos fazem um uso reduzido da maioria das ferramentas de aprendizagem colaborativa, que possibilitariam maior interação com seus colegas, apesar da distância física, característica do ensino semipresencial. Uma exceção é o alto uso de fóruns, pois esta ferramenta está destinada principalmente às atividades relacionadas ao seu curso.

A frequência de uso destinada às ferramentas da internet que possibilitariam interações, apesar de baixa, demonstra um potencial para uso de redes sociais como o Facebook. 
Mesmo utilizando as ferramentas preferencialmente para as atividades da graduação, observa-se que, aos poucos, os alunos buscam explorar as demais potencialidades das ferramentas da internet. Isso permite sugerir que estratégias que fomentem o uso desses recursos devem ser buscadas como, por exemplo, a implantação de uma Comunidade Virtual de Aprendizagem direcionada ao ensino das Ciências Biológicas.

Por meio do perfil aqui descrito, é possível compreender como os licenciandos em Ciências Biológicas utilizam as ferramentas da internet para aprender e estudar, obtendo-se um referencial para a formulação de estratégias potencializadoras do uso de ferramentas colaborativas direcionadas a alunos de educação a distância ou semipresencial. Assim se pretende construir métodos que permitam a construção de abordagens para aprendizagem colaborativa que incentivem a aprendizagem de conteúdos além daqueles disponibilizados pelo curso de graduação.

\section{Agradecimentos}

Neusa H. S. P. Martins recebe uma bolsa de mestrado pela CAPES. Este estudo foi realizado com recursos da Fundação Carlos Chagas Filho de Amparo à Pesquisa do Estado do Rio de Janeiro (FAPERJ - E-26/110.546/2014) e do Conselho Nacional de Pesquisa (CNPq - 481714/2013-8).

\section{Referências bibliográficas}

Bianconi, M.,\& Caruso, F. (2005). Educação não formal. Ciência E Cultura, 57(4), 2020. Disponível em: http://cienciaecultura.bvs.br/scielo.php?pid=S0009$\underline{67252005000400013 \& \text { script }=\text { sci arttext }}$

Bransford, J. D., Brow, A. L.,\& Cocking, R. R. (2007). Como as pessoas aprendem: cérebro, mente, experiência e escola. (p. 384). São Paulo: SENAC São Paulo.

Brown, S. A. (2012). Seeing Web 2.0 in context: A study of academic perceptions. The Internet and Higher Education, 15(1), 50-57. doi:10.1016/j.iheduc.2011.04.003 
CONAE Conferência Nacional de Educação (2010). Construindo o Sistema Nacional Articulado de Educação: o Plano Nacional de Educação, diretrizes e estratégias; Documento Final. Brasília, DF. Disponível em: http://conae.mec.gov.br/images/stories/pdf/pdf/doc base documento final.pdf

Cormode, G., \& Krishnamurthy, B. (2008). Key differences Web 1.0 and Web 2.0. First Monday, 13 (6). http://dx.doi.org/10.5210/fm.v13i6.2125

Dillenbourg P. (1999) What do you mean by collaborative learning?. In P. Dillenbourg (Ed) Collaborative-learning: Cognitive and Computational Approaches. (pp.1-19). Oxford: Elsevier. Disponível em: http://tecfa.unige.ch/tecfa/publicat/dil-papers2/Dil.7.1.14.pdf

FNDE Fundo Nacional de Desenvolvimento da Educação. (2010). Resolução No 17, De 10 de Junho de 2010. Acesso em 3 de novembro de 2014. Disponível em: https://www.fnde.gov.br/fndelegis/action/UrlPublicasAction.php?acao=abrirAtoP ublico\&sgl tipo $=$ RES\&num ato $=00000017 \&$ seq ato $=000 \& v \mid r$ ano $=2010 \&$ sgl or gao $=\mathrm{CD} / \mathrm{FNDE} / \mathrm{MEC}$

Fraenkel, J., \& Wallen, N. (2008). How to design and evaluate research in education. (7th ed., p. 704). New York: McGraw-Hill.

Gialamas, V., Nikolopoulou, K., \& Koutromanos, G. (2013). Student teachers' perceptions about the impact of Internet usage on their learning and jobs. Computers \& Education, 62, 1-7. doi:10.1016/j.compedu.2012.10.012

Hussain, I. (2012). A Study to Evaluate the Social Media Trends among University Students. Procedia - Social and Behavioral Sciences, 64, 639-645. doi:10.1016/j.sbspro.2012.11.075

O'reilly, T. (2007) What is Web 2.0: design patterns and business models for the next generation of software. Communications \& Strategies, First Quarter, 1, 17-37 Disponível em: http://ssrn.com/abstract=1008839

Resolução CNE/CP no 1/2002 (2002). Institui Diretrizes Curriculares Nacionais para a Formação de Professores da Educação Básica, em nível superior, curso de licenciatura, de graduação plena. Diário Oficial da União, Brasília, DF. Disponível em: http://portal.mec.gov.br/cne/arquivos/pdf/CP012002.pdf 
Rolando, L. G. R., Salvador, D. F., \& Luz, M. R. M. P. (2013). The use of internet tools for teaching and learning by in-service biology teachers: A survey in Brazil. Teaching and Teacher Education, 34(1), 46-55. doi:10.1016/j.tate.2013.03.007

Stahl, G., Koschmann, T., \& Suthers, D. (2006). Computer-supported collaborative learning: An historical perspective. In R. K. Sawyer (Ed.), Cambridge handbook of the learning sciences, 19, 409-426. Cambridge: Cambridge University Press. Disponível em: http://gerrystahl.net/cscl/CSCL Portuguese.htm

Ullrich, C., Borau, K., Luo, H., Tan, X., L., S., \& Shen, R. (2008). Why web 2.0 is good for learning and for research: principles and prototypes. In 17th International World Wide Web Conference, Beijing: China, (pp. 1-10), Beijing, China. Disponível em: http://dl.acm.org/citation.cfm?id=1367593

Yapici, İ. Ü., \& Hevedanli, M. (2012). Pre-Service Biology Teachers' Attitudes towards ICT using in Biology Teaching. Procedia - Social and Behavioral Sciences, 64, 633638. doi:10.1016/j.sbspro.2012.11.074 


\section{APÊNDICES - QUESTIONÁRIO DE USO DA INTERNET}

1- Você utiliza sites de busca na internet (Google, Ask, Altavista entre outros) para estudar e aprender Biologia?

2- Caso você utilize sites de busca na internet para estudar e aprender Biologia, descreva como você os utiliza para essa finalidade.

3- Você utiliza e-mail para estudar e aprender Biologia?

4- $\quad$ Caso você utilize e-mail para estudar e aprender Biologia, descreva como você o utiliza para essa finalidade.

5- Você utiliza chat (MSN, Gtalk, entre outros) para estudar e aprender Biologia?

6- Caso você utilize chat para estudar e aprender Biologia, descreva como você o utiliza para essa finalidade.

7- Você utiliza o Twitter para estudar e aprender Biologia?

8- Caso você utilize o Twitter para estudar e aprender Biologia, descreva como você o utiliza para essa finalidade.

9- $\quad$ Você utiliza o Skype para estudar e aprender Biologia?

10- $\quad$ Caso você utilize o Skype para estudar e aprender Biologia, descreva como você o utiliza para essa finalidade.

11- Você faz download de arquivos da internet para estudar e aprender Biologia?

12- Caso você faça download de arquivos da internet para estudar e aprender Biologia, de quais tipos de arquivo você faz download para essa finalidade?

13- Você utiliza imagens da internet para estudar e aprender Biologia?

14- Caso você utilize imagens da internet para estudar e aprender Biologia, descreva como você as utiliza para essa finalidade.

15- Você utiliza vídeos da internet para estudar e aprender Biologia?

16- Caso você utilize vídeos da internet para estudar e aprender Biologia, descreva como você os utiliza para essa finalidade.

17- Você utiliza a Wikipédia para estudar e aprender Biologia?

18- $\quad$ Caso você utilize a Wikipédia para estudar e aprender Biologia, descreva como você a utiliza para essa finalidade.

19- Você utiliza o Google Docs para estudar e aprender Biologia?

20- Caso você utilize o Google Docs para estudar e aprender Biologia, descreva como você o utiliza para essa finalidade.

21- Você utiliza blogs para estudar e aprender Biologia?

22- Caso você utilize blogs para estudar e aprender Biologia, descreva como você os utiliza para essa finalidade.

23- Você utiliza fóruns para estudar e aprender Biologia?

24- Caso você utilize fóruns para estudar e aprender Biologia, descreva como você os utiliza para essa finalidade.

25- Você utiliza o Facebook para estudar e aprender Biologia?

26- Caso você utilize o Facebook para estudar e aprender Biologia, descreva como você o utiliza para essa finalidade.

27- Você utiliza o Orkut para estudar e aprender Biologia?

28- Caso você utilize o Orkut para estudar e aprender Biologia, descreva como você o utiliza para essa finalidade.

29- Você utiliza o Google+ para estudar e aprender Biologia?

30- Caso você utilize o Google+ para estudar e aprender Biologia, descreva como você o utiliza para essa finalidade.

31- Você utiliza o Ning para estudar e aprender Biologia? 
32- Caso você utilize o Ning para estudar e aprender Biologia, descreva como você o utiliza para essa finalidade.

33- Você utiliza o Second Life para estudar e aprender Biologia?

34- Caso você utilize o Second Life para estudar e aprender Biologia, descreva como você o utiliza para essa finalidade. 